\title{
Voting Originated Social Dynamics: Quartile Analysis of Stochastic Environment Peculiarities
}

\author{
V. M. Maksimov ${ }^{*, a}$ and P. Yu. Chebotarev**,b \\ * Moscow Institute of Physics and Technology, Dolgoprudny, Russia \\ ** Trapeznikov Institute of Control Sciences, Russian Academy of Sciences, Moscow, Russia \\ e-mail: ${ }^{a}$ vladislav.maksimov@phystech.edu, ${ }^{b}$ pavel4e@gmail.com \\ Received December 16, 2019 \\ Revised April 29, 2020 \\ Accepted May 25, 2020
}

\begin{abstract}
The model of voting originated social dynamics in a stochastic environment (the ViSE model) is considered. Within this model, the influence of the heaviness of distribution tails on the effectiveness of egoistic and altruistic strategies in terms of maximizing two criteria, the average capital increment and the number of non-bankrupt participants, is investigated. Homogeneous societies and four types of distributions used to generate proposals (Gaussian, logistic, Student's with 3 degrees of freedom, and symmetrized Pareto distributions) are studied. To assess the effect of tail heaviness, all distributions are unified by quartile using scatter. Such an approach can be used to compare the heavy-tailed distributions that are commensurable by density with other distributions under consideration on an interval containing $90 \%$ of observations.
\end{abstract}

Keywords: ViSE model, social dynamics, dynamic voting, stochastic environment, pit of losses, egoism, altruism, heavy-tailed distributions

DOI: $10.1134 /$ S0005117920100069

\section{INTRODUCTION}

The main assumptions of the ViSE (Voting in Stochastic Environment) model, suggested for analyzing the utility of collective decisions, are as follows [1, 2].

Society initially consists of $n$ participants (also called agents). Each participant is characterized by the current value of his capital (debt, if negative), or utility. The vector of nonnegative initial capitals of all participants is given. In each step $m=1, \ldots, M$, a proposal is put to the vote, and the participants vote, guided by certain strategies, for or against it. A proposal is the vector of algebraic capital increments of all participants. The proposals approved through an adopted voting procedure are implemented. One of the variants of the model assumes the elimination of the bankrupt participants, i.e., the participants whose capital has become negative (the extinction mode). The dynamics of the participants' capital can be analyzed to compare voting strategies and collective decision procedures, in order to choose the optimal ones among them in terms of maximizing an appropriate criterion.

By assumption, each proposal is generated in a stochastic way: its components are the realizations of random variables, in the simplest case, independent and identically distributed, with a given mean $\mu$ and a given standard deviation $\sigma$. Therefore, the proposals put to the vote can also be called the proposals of a stochastic environment, or the proposals of the environment. This environment is favorable if $\mu>0$; neutral if $\mu=0$; and unfavorable if $\mu<0$.

Let $\boldsymbol{c}(m)$ be the vector of the participants' capitals at the end of step $m \geq 0$, and also let $\boldsymbol{c}(0)$ be the vector of initial capitals. In the extinction mode, these participants include only those whose 
capital at the end of step $m$ is nonnegative. In the non-extinction mode, the number of components of the vector $\boldsymbol{c}(m)$ remains equal to $n$. Then

$$
\boldsymbol{c}(m)=r(\boldsymbol{c}(m-1)+\boldsymbol{\zeta}(m) I(m)), \quad 0<m \leq M,
$$

where $\boldsymbol{\zeta}(m)$ is the environment proposal vector in step $m ; M$ denotes the total number of proposals;

$$
I(m)= \begin{cases}1 & \text { if the proposal } \boldsymbol{\zeta}(m) \text { is approved through voting } \\ 0 & \text { otherwise; }\end{cases}
$$

$r(\cdot)$ is the operator eliminating all negative components from a given vector (the extinction mode) or the identity operator (the non-extinction mode).

The effectiveness of individual voting strategies and collective decision procedures is compared by means of analyzing the statistical regularities of changes in the capital vector (1) in different environments, i.e., for different distributions used to generate proposals.

This paper examines the decisions made by simple majority voting, and the following principles of voting by participants. An egoist supports a proposal if and only if it increases his capital. Altruists are supposed to have the following strategy. All participants are arranged in the ascending order of the current capital value. A number $\delta \in\left[\frac{1}{n}, 1\right]$ is fixed, and for the current proposal of the environment the sum of capital increments of the poorest ${ }^{1}[\delta n]$ participants is calculated, where $[\cdot]$ denotes the integral part operator. An altruist supports a proposal if and only if this sum is positive. The support window of altruists is the segment $[0, \delta]$, where $\delta$ can be expressed in percentage terms.

In [2], for several types of stochastic environments, the influence of social attitudes (egoism, altruism) on the dynamics of social welfare was investigated. To analyze the influence of the type of distribution, it is necessary to unify the distributions by the position and scale parameters. The proposals were generated by means of symmetric distributions; hence, the median was used as the position parameter (which actually coincided with the mean, since it existed for the distributions under consideration). The scale was estimated by the standard deviation, but it was concluded that this approach leads to not entirely natural results, i.e., to the comparison of distributions that are radically different on the interval where almost all probability is concentrated. Therefore, it was emphasized that a new study using a scale measure more adequate to the problem under consideration should be carried out.

This program will be implemented below. In Sections 2 and 3, the ViSE model and the disadvantages of unifying distributions by variance are discussed. In Section 4, the foundations of a different approach to the unification of distributions (by quartiles) are given. In Section 5, this approach is applied to analyze social dynamics in the case of proposals generated using the Gaussian distribution and symmetrized Pareto distributions. In Section 6, the logistic distribution and Student's $t$-distribution are also included in comparison. In Sections 5 and 6, some qualitative conclusions regarding the influence of social attitudes of different participants and the type of stochastic environment on the capital dynamics and the rate of bankruptcy are formulated. Obtaining these conclusions is actually the main goal of the paper. The study presented below involves simulation modeling, since the analytical methods developed in [1] yield no final expressions for the quantities of interest for the participants with altruistic strategies. In Section 7, the main results of this paper are summarized.

\footnotetext{
${ }^{1}$ After the first approved proposal, the capitals of participants can be considered different, since the proposals are generated by random variables with a continuous distribution. If in the initial state the capitals do not differ, then before the approval of the first proposal, the altruists find the sum of capital increments of the participants numbered $1, \ldots,[\delta n]$.
} 


\section{ViSE MODEL: FOUNDATIONS AND CONNECTION WITH REALITY}

The ViSE model is an extension of the voting model proposed by A.V. Malishevskii; for example, see the book [3], Chapter 2, Section 1.3. Malishevskii was the first to show that a person with a monopoly right to put proposals to the vote (further called "proposer") can, in fact, pass any decision through the voting body in several steps. In the model, each voter supports any proposal that increases his individual utility, while the voting procedure is qualified majority, but not unanimity. To achieve his goal, the proposer uses the algorithm of "small handouts to the majority." Each proposal that he puts to the vote is beneficial to a certain majority sufficient to make a decision, but gives this majority in total less than it takes from the minority. Clearly, if everyone is in the minority at least once, then the cumulative result of a series of such proposals can be made arbitrarily disadvantageous for all voters, so unbeneficial that the proposer's original solution will be unanimously approved.

How to deal with this paradox, which demonstrates the vulnerability of democratic procedures? A possible approach is to eliminate it by making the model more rigid. In the original model, the proposer has tremendous freedom: he has access to any proposals from the space, the dimension of which is equal to the number of voters. In practice, proposals usually belong to a lower-dimension space. Will the paradox persist if the dimension of the proposal space is, for example, 2 or 3 ? In accordance with the research works of C. Plott, R. McKelvey, N. Schofield, D. Saari and others [4-8], in many cases it will remain, although it is necessary to fulfill certain conditions, in particular, the ones connecting the majority threshold with the dimension of the proposal space.

Well, how democracy can be rehabilitated? Quite obviously, it is necessary to restrict the proposer's monopoly or change the voting strategies. ${ }^{2}$ For example, suppose the voters can make proposals themselves. But in what order? Simultaneously (so that there is a choice of several projects) or alternately? Alone or in groups? Are alliances permanent or situational? Are there external proposers and how many? What information do the participants have about the proposals and strategies of others? Can strategies be constructed reflexively? Depending on the details of the procedure, the scope of the rights of the participants and their awareness, there are many gametheoretic statements and ... a rather chaotic situation. A number of problems of this kind can be solved [9-14], but the results are heterogeneous and local. All these lead to a mosaic rather than a general system of regularities. The reason is mainly that the above-mentioned statements do not form a hierarchical structure, a tree. There is no basic "zero" problem statement, after the study of which it would be possible to move along the side branches to more and more sophisticated statements.

The ViSE model is a possible zero of this kind. This model includes no proposer with his own strategy: there is a favorable, neutral or unfavorable environment instead, endowed with the prerogative to propose. The variety of proposals is ensured by its stochastic nature. In the simplest case, the environment is characterized by favorability, the ratio $\mu / \sigma$, where $\mu$ is the mean value of the individual proposal and $\sigma$ is the standard deviation. The latter can be considered as a scale, a unit of measurement for $\mu$. The most natural distribution in the zero problem statement is the Gaussian one.

Will the Malishevskii paradox persist? In other words, could a series of decisions taken by the majority be ultimately disadvantageous to everyone? Disadvantageous not sometimes, but under certain conditions, systematically, "as a rule."

This question is fundamental, since it concerns the very nature of collective decisions, and not the external manipulation of them. In an alternative way, it can be formulated as follows: does voting

\footnotetext{
${ }^{2}$ In the voting models under consideration, a strategy is understood as an algorithm following which an agent uses his available information for making individual decisions on the support of proposals or candidates.
} 
serve as an effective filter in the interaction of society with an unfavorable (but not extremely inventive) environment that requires binary solutions from it?

This question is not trivial, and the answer in the zero problem statement is as follows [15]: yes, the "pit of losses" paradox (a variant of the Malishevskii paradox) is realized in a moderately unfavorable environment. More specifically, decisions made by majority voting are usually disadvantageous to society.

Without continuing to discuss this topic in detail, consider now some real-life prototypes of the ViSE model. Imagine a country where decisions are made by the parliament, further called the State Duma. The proposals come from an environment that is external to it. For example, $\mathrm{OPEC}+$ proposes an agreement to limit oil production. Some actors inside the country are ready to support this proposal, others are not. For some political forces (in particular, for the lobbying ones) represented in the State Duma, the proposal is beneficial; for others, it is not. And it has to be approved or rejected. Another example: the United States or the North Atlantic Treaty Organization (NATO) are proposing to sign a new arms control agreement. This situation is the same as the one described above. Or a decision of the Hague District Court orders to pay compensation to the families of the victims of Malaysia Airlines flight MH17, crashed in eastern Ukraine. Different political forces hold different opinions. Or there may be a proposal to participate in a new agreement to limit greenhouse gas emissions. If the foreign policy situation is favorable for the country, then there will be more proposals beneficial to it; if not, then vice versa.

Further, since decisions are made by the State Duma, proposals coming from the presidential administration or from the government are also external to it. And they reflect a definite external situation: first, to what extent the president and/or the government act in the interests of the country; second, these proposals inevitably translate general economic, social, and political trends and challenges. As relevant examples, consider a proposal for amendments to the Constitution of the Russian Federation, or a proposal for the introduction of emergency measures in connection with the coronavirus pandemic. The general situation affects the country in addition to the decisions of the State Duma, but the subject of study is not this unconditional impact, but the effectiveness and rationality of collective decisions.

Here is another class of examples. Imagine the board of directors of a company. The top management or planning department proposes a new development strategy for the company. It is necessary to approve or reject it, sending back for revision (for the time being, following the same course as before). In addition, each member of the board of directors has his own opinion and interests. "Games" with the top management or planning department of the company can be considered, but there is another important set of issues related to the effectiveness of the voting procedure and the strategies of its participants. Or another company, a potential partner, proposes a deal to this company. In some ways the deal is beneficial, in others it is not. The proposal has to be approved or rejected. And if it was not rejected absolutely, it can be sent back for revision. Moreover, the better the company is doing (and the better the economic situation is), the more favorable the environment behaves to it and the more advantageous proposals on average are generated by the environment (potential partners) for voting.

Is it reasonable to assume that the components of a complex proposal related to different agents are statistically independent? Since the proposals are quite heterogeneous, the benefit of a proposal for political forces (or board members) $A$ and $B$ does not imply either the advantage or disadvantage of this proposal for political force $C$. As is well known, in politics, unexpected alliances can arise for various reasons: the Left with the Ultra-right, etc. Therefore, independence is an admissible zero statement. As an opposite example, consider a class of situations in which the payoffs of different forces have a fixed sum or are strongly connected in another way. For this class of situations, the corresponding additional constraints can be imposed within the model. 
Does the ViSE model yield any conclusions applicable to reality? Here are some examples.

1. The model implements [16] the well-known "small party effect": in the presence of two large parties, neither of which has a majority, a small party forming a majority with any of the large ones gains an advantage; sometimes, when large parties take opposite positions, such a small party can gain a position comparable to a dictator.

2. The ViSE model reveals [16] one of the stability mechanisms of the bipartisan system with almost equal parties by their power.

3. The model implements $[16,17]$ the mechanism of "the snowball of cooperation": since belonging to a group is usually more beneficial than protecting one's own interests individually, participants join the group, and as it grows, group egoism becomes closer to altruism.

4. For a long time, the power indexes of factions in a parliament have been a classical analysis tool in politics. The study of the dependence of utility increments in the ViSE model and power indices leads to the following conclusion: for the simplest set of parameters, the average utility increments are linearly related (after natural normalization) to the values of the Banzhaf index. This fact, on the one hand, gives a new interpretation for the utilities in the ViSE model; on the other hand, allows selecting the Banzhaf power index among the variety of power indexes as the one having "external" interpretation and confirmation. Thus, the Banzhaf influence model and the ViSE model reinforce each other. (This is a conclusion of an unpublished paper).

5. The ViSE model leads to an important result discussed above, the "pit of losses" paradox. This paradox has a mechanism [15] that is undoubtedly implemented in practice. More specifically, the payoffs are on average smaller and the losses are larger in an unfavorable environment than in a neutral environment with the same scatter; thus, the average difference between the absolute values of a payoff and a loss is negative. Therefore, the proposals accepted by simple majority voting with a small margin of votes in favor will more often yield a total loss than a total gain. This mechanism is hardly revealed by other models. A practical implication is as follows: the less favorable the environment is, the higher the voting threshold for new proposals should be.

6. The conclusion of item 5 (see above) has exceptions associated with heavy-tailed distributions, which in some cases adequately describe political and economic uncertainty. This range of issues was studied by the members of the authors' research team: the concept of the optimal voting threshold was introduced in [15], and its general formula was expressed in [18].

7. The comparison of light- and heavy-tailed distributions as generators of uncertainty in the ViSE model with altruistic strategies with different support windows (see [2]) revealed some effects correlated with the phenomenon of antifragility and the barbell strategy discussed by N. Taleb.

8. In addition to the pit of losses, analysis of the ViSE model reveals another interesting paradox of voting: in a really favorable environment, under certain conditions, majority voting can give worse, on average, results for society than accepting all proposals without any consideration. Numerically, this paradox is symmetric to the pit of losses paradox, which seems at first glance to be a simple fact, but has a nontrivial proof.

9. One of the key problems, not only in social sciences but also in social practice, is to compare the rationality of social strategies (from the standpoint of an individual and society), i.e., the narrowly selfish and prosocial ones, including collectivism, focused on the common welfare, involving philanthropy, etc. A possible goal of such research is to show the citizen that he will not lose by adhering to prosocial strategies; or rather, to find out what he will obtain using different strategies in different conditions. Traditionally, the problems of this kind are numerically analyzed on the basis of matrix games. But the totality of the results obtained, like in the strategic voting problems, resembles a mosaic rather than general regularities. The ViSE model provides 
an extraordinary flexibility in setting external conditions, the structures of society and social attitudes. For example, it can describe a rich variety of prosocial strategies, and the conclusions from the analysis will be in the form of regularities. It is possible to correlate these conclusions with reality, as well as to assess how vital the model mechanisms leading to them are. This application of the ViSE model is more extensive than the ones listed previously. Therefore, it will be considered separately; see the detailed discussion below.

Well, as noted above, the phenomena of cooperation, collectivism and altruism are often studied by analyzing the dynamics of simple games using simulation modeling and "laboratory" experiments with people. The Prisoner's Dilemma is one of the most popular games of this class.

However, the metaphorical conditionality of this approach is quite obvious. Real-life situations in which a person chooses a line of social behavior are not so often like the Prisoner's Dilemma. Its key condition is the lack of information: the player does not know which strategy his opponent will choose, and this is what determines his payoff/loss. If the other player also extends his hand to him, both will win compared to the individualism of each; if he refuses, then the one who refused will "break the bank," and the one whose hand hangs in the air will lose. The peculiarity of real life is that people can negotiate ${ }^{3}$ and control further compliance with the agreements. That is, the key element of this model does not always correspond to reality. In addition, the Prisoner's Dilemma is a game of two persons, whereas a larger number of agents are usually involved in real-life cooperation and its mechanism is not reduced to pairwise interactions. Despite that only a small share of actual cooperation problems are adequately described by the models based on the Prisoner's Dilemma (and in other cases, the connection of these models with reality is only metaphorical), these models are valuable and interesting. They touch upon an important aspect of reality, i.e., the influence of information uncertainty on the cooperation of two persons, and non-trivial results can be obtained within their framework.

This restriction of the Prisoner's Dilemma as a model of cooperation, called pairwise interactions, can be removed by considering other games, such as, for example, Public Goods. But here, the participant's benefit completely depends on the relationship between his strategy and the strategies of other players unknown to him, and the free rider problem (parasitism) is at the center of analysis.

In real life, there is also free riding, which complicates cooperation, but the problems of cooperation are not limited to this phenomenon.

The problem of a real person, who determines the degree of cooperativeness for his behavior, is mainly not ignorance of what others will do, but that "self comes first." for himself and his children, and putting them on will make them happy. Or he can invest his money or efforts in a more or less abstract public good, but his children will continue to wear old shirts. Public good will most likely bring benefits to everyone over time, or to those who are unprotected even today, but the optimal proportion of investments in the public and individual good is different for everyone and depends on the circumstances.

What is important to emphasize, in real life, even adequate information about the behavior of other agents (often available) does not eliminate the complexity of choosing an appropriate strategy. This complexity consists in that the effect of investments in public good is delayed and difficult to predict. Because, figuratively speaking, agents sail on a common ship (or on different boats) on a stormy sea, where various surprises await them. The new shirt is already useful for each agent, but what and when he will obtain by investing in the public good is difficult to assess. Thus, the source of ambiguity is not only (and often not so much) the unknown strategies of the "colleagues," but also the uncertainty of the environment. (Of course, not to mention the difficult problem of discounting.)

\footnotetext{
3 This is also neglected in such games as The Ultimatum and The Dictator.

${ }^{4}$ In Russian, this proverb sounds as follows: "one's own shirt is closer to the body."
} 
As a matter of fact, precisely this environment is one of the main elements of the ViSE model. A participant can invest his resource (vote in the case of voting procedures) in his good or in the good of the entire society or any of its strata, about which he cares (but not necessarily belongs to, for example, the social group of the poorest). In addition, he can have an arbitrarily complex ("combined") voting strategy, with different weights taking into account his good, the good of the entire society and the good of any number of groups. This gives rich flexibility in defining behavioral strategies that are structurally similar to those in the real world. For example, combined strategies can be used to model a social stratum called responsible elite [19]. Including combined utility functions in game-theoretic models like Public Goods don't seem quite as natural.

As has been noted above, the ViSE model with altruistic agents can be analyzed to formulate certain regularities. Here are some of the simplest conclusions [2, 19, 20]. First, the altruism of principle (or the "hard-core" altruism, in the terminology of Edward Wilson [21]), which is a relative rarity, can in some cases be replaced by a rational strategy based on the theoretically confirmed advantage of collectivism, which can lead to the perception of an increasing part of society as a one's own collective. Second, generally speaking, society does not need the majority of its members to be altruists: the presence of a very moderate share of them leads to a comparable result. In particular, a relatively small altruistic faction can protect society from bankruptcy, but this faction itself is in the most vulnerable position. Third, if the altruistic faction reaches a certain critical mass, its vulnerability is reduced to acceptable limits. Fourth, the utility of certain altruistic strategies for society strongly depends on the properties of the environment: for example, in a favorable environment, charity is rational in relation to a relatively few, but in an unfavorable environment it is more correct to help everyone, since everyone is at risk. And fifth, since it is of high utility for society to have participants with a prosocial voting strategy, but these participants themselves are the least protected, it makes sense for society to provide additional support to these participants; in addition, the benefits to others are higher than their support costs.

The theoretical mechanisms of implementing these and other conclusions can be traced by studying the ViSE model. These mechanisms can be correlated with reality, which makes it possible to assess the adequacy of the conclusions to certain real-life situations.

The last (5th) conclusion determines the approach to the comparison of taxation schemes. Therefore, the following item can be added to the list of applications of the model.

10. The ViSE model can be docked with financial models of taxation distributed in favor of participants working for society. Using game-theoretic models of cooperation for the same goal seems less productive.

For applying mathematical models, a common element is to perform identification, i.e., to determine parameter values matching available observations.

The ViSE model can be, in principle, subjected to identification. For example, consider the board of directors of a company or parliament represented by different forces. It is possible to estimate the parameters $\mu$ and $\sigma$ and the type of the generating distribution by assessing the benefits of a series of projects put to the vote for individual voters/political parties. In turn, this will allow one to find an optimal voting threshold for proposals of the type under study, as well as to assess the dynamics of utilities and the severity of various effects.

However, like in the case of several game-theoretic models, the application of the ViSE model does not reduce to the calculation of parameters and effects by observations. The revelation of phenomena and mechanisms that are difficult to observe without the model is also valuable. One of the simple examples, already discussed above, is a diverse minority that systematically loses more than the majority gains. This is a fundamental and very important phenomenon that can be called the main vice of voting. It must always be borne in mind for any collective decision-making, and it 
requires the application of specific measures that can first be tested on models. And an example of a mechanism that can be used in practice is the "snowball of cooperation," i.e., the approximation of the collective egoism of a successful and open group (welcoming new members) to altruism. This metaphor was used in the paper [22], where the mechanisms of the development of civil society were discussed on the basis of the ViSE model; more specifically, the issues of building a mutual support network of the cells of civil society were considered. In this case, the decision to support any proposal is made by each cell independently, but those cells that systematically refuse to support others are excluded from the network. In the above list of applications (in particular, see item 9), some other phenomena and mechanisms have been also mentioned. They can be correlated with reality, by assessing their effectiveness in specific conditions.

\section{UNIFYING DISTRIBUTIONS BY VARIANCE FOR ViSE MODEL ANALYSIS}

Among other stochastic environments, the paper [2] considered the environments generating proposals that obey the symmetrized Pareto distribution (SP-distribution) with superheavy tails.

The SP-distribution with parameters $k>0, a>0$, and $\mu$ (see [2]) has the density function

$$
f(x)=\frac{k}{2 a}\left(\frac{|x-\mu|}{a}+1\right)^{-(k+1)}, \quad x \in \mathbb{R},
$$

the mean $\mu$ if $k>1$, and the variance $\sigma^{2}=2 a^{2} /((k-1)(k-2))$ if $k>2$. If the above inequalities are not satisfied, the corresponding integrals diverge. As $k \rightarrow \infty$ the SP-distribution converges to the Laplace distribution with the density function $f(x)=\frac{1}{\sigma \sqrt{2}} \exp \left(-\frac{|x-\mu| \sqrt{2}}{\sigma}\right)$; for details, see Lemma 3 of the paper [2].

In [2], the average values of the participant's capital for one voting step were compared for several symmetric distributions with the same mean $\mu$ and variance $\sigma^{2}$. It was noted that two symmetric distributions with identical values of the parameters $\mu$ and $\sigma$ may differ dramatically. For example, consider the distributions whose densities are demonstrated in Fig. 1; apart from their continuity, symmetry, infinity, and common mode, one would hardly observe any other commonality. At the point of the mode $x=1$, their densities are related as 20 to 1 ; at the point $x=0$, as 1 to 43 .

From Fig. 1 it is difficult to imagine that these two distributions have equal variance: as estimated by eye, the typical deviations from $\mu=1$ for the distribution with $k=1000$ are much higher. The equality of their variances can be explained using Fig. 2, where these two density functions (for $x \geq 1$ ) are shown in logarithmic scale. The tails of the distribution with $k=2.01$, seeming light

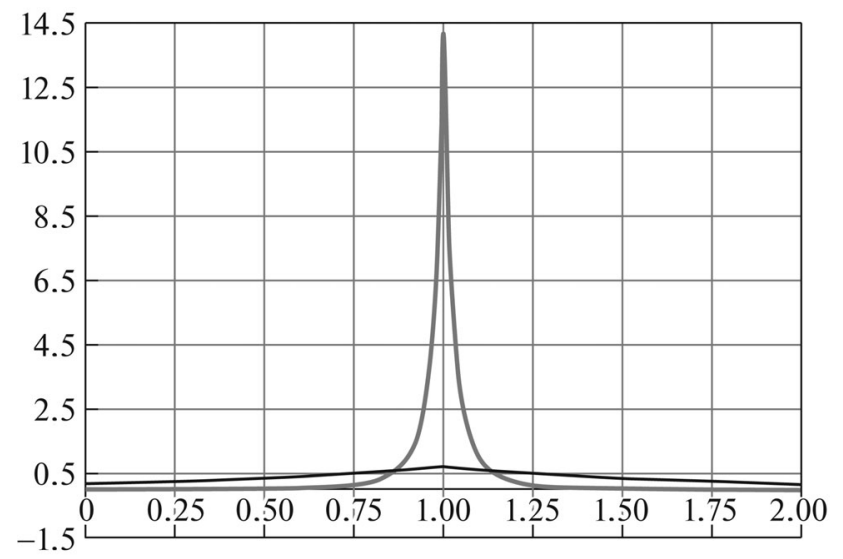

Fig. 1. Density functions of two symmetrized Pareto distributions with $\mu=1$ and $\sigma=1: k=2.01$ (grey line) and $k=1000$ (black line).

AUtOMATION AND REMOTE CONTROL Vol. $81 \quad$ No. 102020 


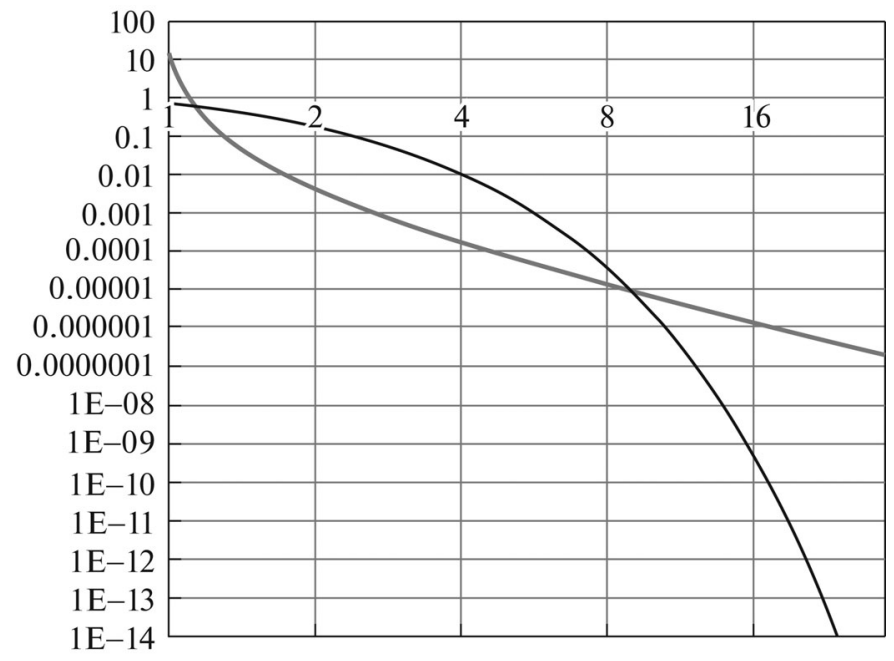

Fig. 2. Density functions of two symmetrized Pareto distributions with $\mu=1$ and $\sigma=1$ in logarithmic scale in case $x \geq 1: k=2.01$ (grey line) and $k=1000$ (black line).

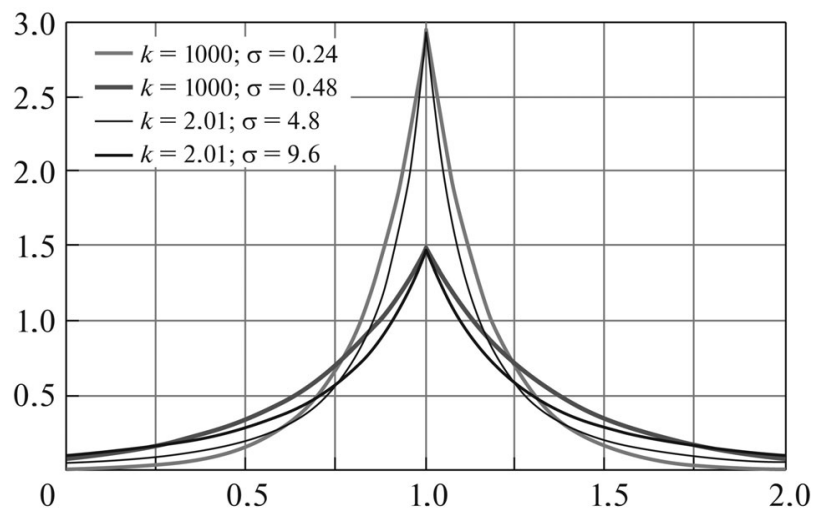

Fig. 3. Examples of density functions of symmetrized Pareto distributions with $\mu=1$.

within the limits of Fig. 1, turn out to be significantly heavier than those of the distribution with $k=1000$ at a large distance from the mean (for $|x-\mu|>8$ ). Since $P(|x-\mu|>8) \approx 7.4 \times 10^{-5}$, the observations relating to such distant tails are extremely rare. The effect of the relative heaviness of these tails in small-scale experiments can be described in terms of the difference between the concepts of "almost impossible" and "practically impossible."

However, thanks to these tails, the SP-distribution with $k=2.01$ has unit variance, like the Pareto distribution with $k=1000$; see Fig. 1 . Thus, the tails weakly affecting the experimental results (in any case, when investigating the ViSE model) make to unify the SP-distribution with $k=2.01$ with other (dissimilar) distributions, if the distributions are unified by $\mu$ and $\sigma$. This leads to a need for a different principle of establishing a correspondence between distributions. Such a principle will be discussed in Section 4.

The controversy in this context of the correspondence established by the mean and variance was noted in [2]: "The present paper analyzes the ViSE originated social dynamics with distributions of different types, but the same mean and variance. This standardization of parameters is rather common and seems to be natural. However, let us return to Fig. 3 [numbering is in accordance with this paper] showing two pairs of SP-distributions which are, in each pair, relatively close in a neighborhood of $\mu$. As was noted, the standard deviations $\sigma$ of these "roughly similar" distributions differ by a factor of 20. If we transform the distributions with super-heavy tails $(k=2.01)$ in 
such a way that they acquire the same variance as the distributions with $k=1000$, then the former distributions contract 20 times and lose all resemblance to the latter distributions in the neighborhood of $\mu$. The question is: which distribution with $k=2.01$ should be considered to be similar to the corresponding distribution with $k=1000$ ? The initial one behaves rather similarly in the central zone, but its variance is 400 times larger. The transformed one has the same mean and variance, but its behavior in the neighborhood of $\mu$ is completely different. There are strong arguments for the first answer. This fact indicates the desirability of an alternative approach to the standardization of distributions. Say, one can consider two symmetric distributions to be "similar" when they have the same $\mu$ and the same interval (centered at $\mu$ ) containing a certain essential proportion of probability. In this case, [...] the conclusions of the present research will be complemented with new ones."

The fact that variance-based standardization over-concentrates heavy-tailed Pareto distributions near the mode is because any increase in the probability of large outliers (taken into account in the calculation of variance quadratically) must be balanced by a decrease in the probability of deviations in the mean range exceeding $\sigma$, and/or by an increase in the probability of deviations less than $\sigma$. Thus, the weighting of the tails under a fixed variance accelerates the decrease in the density function for higher distances to the mean (coinciding with the mode) and, possibly, enlarges the density function at the mode itself.

Within the framework of the ViSE model, this paper compares with each other several symmetric continuous distributions with the same mean $\mu$ and an equal width of the interval centered at $\mu$ that contains "half" of the distribution. (On this interval, the integral of the density function is 0.5.) This approach will be called the standardization of symmetric distributions by quartiles, or by the mean and one-sided median.

\section{STANDARDIZATION OF DISTRIBUTIONS BY QUARTILES FOR VISE MODEL ANALYSIS}

In the study of the ViSE model, it is of great interest to analyze the influence of the type of distribution used to generate proposals on social dynamics. The distributions under consideration are unimodal and symmetric with respect to the mode. In this case, the notion of the type of distribution includes, in particular, the weight of tails and the form of the density function near the mode, but excludes $[23,24]$ the parameters of position (the mean, coinciding here with the mode and median) and scatter/scale (the variance). For symmetrized Pareto distributions [2], the type of distribution is defined by the parameter $k>0$ : the smaller $k$ is, the heavier the distribution tails will be. For $k \leq 2$, the Pareto distribution has no variance, and for $k \leq 1$ it also has no mean.

A measure often used to characterize the heaviness of the distribution tails (and also the shape of its peak) is the kurtosis proposed by K. Pearson: $\gamma_{2}=\frac{\mu_{4}}{\sigma^{4}}-3$, where $\mu_{4}=\mathrm{E}\left[(X-\mathrm{E} X)^{4}\right]$ denotes the fourth central moment of the distribution and $\sigma$ is its standard deviation. However, kurtosis is inapplicable here: for Pareto distributions it exists only if $k>4$, whereas this paper, as well as [2], examines Pareto distributions with $k$ close to 2 . Alternative measures, as a rule, are defined in terms of distribution quantiles; for example, see [23].

To assess the influence of the type of distribution on social dynamics, compare distributions with the same values of the quartiles $Q_{1}, Q_{2}$, and $Q_{3}$, cutting off $1 / 4,1 / 2$, and $3 / 4$ of the unit probability, respectively: $Q_{s}=F^{-1}\left(\frac{s}{4}\right), s=1,2,3$, where $F(\cdot)$ is a continuous distribution function. Such distributions will be considered analogous by mode (due to symmetry and unimodality, the role of mode is played by the median, i.e., the central quartile) and by scale (scatter).

Thus, two symmetric distribution functions $F_{1}(x)$ and $F_{2}(x)$ are included in the same class if

$$
F_{1}^{-1}(0.25)=F_{2}^{-1}(0.25) ; \quad F_{1}^{-1}(0.5)=F_{2}^{-1}(0.5) .
$$






Fig. 4. Density functions of distributions for which $\mu=0$ and $Q_{3} \approx 53.95918$ : Gaussian $(\sigma=80)$, logistic, Student's with 3 degrees of freedom ("3 deg.") and symmetrized Pareto with different $k$.

Due to the symmetry of the distributions, from these equalities it follows that $F_{1}^{-1}(0.75)=$ $F_{2}^{-1}(0.75)$. Note that this method of comparable scaling of distributions was considered most acceptable in $[23,25]$ and a number of other publications.

For the sake of definiteness, consider the classes of distributions including the Gaussian distribution (normal distribution) with the parameter $\sigma=80$. Each such class is characterized by the median $F^{-1}(0.5)$ of all distributions contained in it. Figure 4 presents the density function of the Gaussian distribution with the parameter $\sigma=80$, along with the densities of several other distributions: Student's $t$-distribution with 3 degrees of freedom, the logistic and symmetrized Pareto distributions with four values of the parameter $k$.

The distributions with $k=2.1$ and $k=2.01$ can be called distributions with superheavy tails, since with heavier tails $(k \leq 2)$, the parameters of social dynamics cannot be estimated by averaging the results of simulation modeling due to no variance. In the worst case $k=2.01,90 \%$ of observations fall into the interval $[-281,281]$.

Note that the quartile approach was adopted to develop the family of tail heaviness measures proposed in [25]. These measures estimate a given distribution in relation to its quartiles and stem from Hinkley's measures [26]. The left quantile weight (LQW) and the right quantile weight (RQW) are given by

$$
\begin{gathered}
\operatorname{LQW}_{F}(p)=\frac{F^{-1}\left(\frac{p}{2}\right)+F^{-1}\left(\frac{1}{2}-\frac{p}{2}\right)-2 F^{-1}\left(\frac{1}{4}\right)}{F^{-1}\left(\frac{p}{2}\right)-F^{-1}\left(\frac{1}{2}-\frac{p}{2}\right)} ; \\
\operatorname{RQW}_{F}(q)=\frac{F^{-1}\left(\frac{1}{2}+\frac{q}{2}\right)+F^{-1}\left(1-\frac{q}{2}\right)-2 F^{-1}\left(\frac{3}{4}\right)}{F^{-1}\left(\frac{1}{2}+\frac{q}{2}\right)-F^{-1}\left(1-\frac{q}{2}\right)},
\end{gathered}
$$

where $0<p<0.5<q<1$. In particular, the heaviness of the left and right tails of a distribution are estimated using $\mathrm{LQW}_{F}(0.25)$ and $\mathrm{RQW}_{F}(0.75)$, respectively.

In contrast to the standardization introduced in [2], where the scatter was estimated by the root-mean-square deviation, the standardization (3) allows comparing density functions that are commensurable by values in the neighborhood of the mean, which includes most of the distribution. Hence, it is possible to draw conclusions about the dependence of the dynamic properties of the ViSE model on the type of distributions, which are better interpreted in terms of applications. 


\section{STANDARDIZATION OF DISTRIBUTIONS BY QUARTILES FOR SOCIAL DYNAMICS STUDY}

Now analyze the results of the study of social dynamics in the ViSE model under the standardization of distributions by quartiles. In Fig. 5a, the average capital increment of egoists for one voting step (ACI, see [2]) have no significant "pit of losses" [2] in the case of the SP-distribution (in contrast to the Gaussian distribution). Recall that "the pit of losses" is an interval for $\mu$, where voting on average leads to decisions that are unbeneficial to society. In a society composed of egoists, in the non-extinction mode, the weighting of the tails of the symmetrized Pareto distribution leads to larger values of the ACI for the same $\mu$.

In a society composed of altruists with the support window [0,65\%] (Fig. 5b), if $k$ is large enough (in this case, for $k>2.3$ ), then the ACI graph has a clear pit of losses; as $k$ increases, it shifts to the right. The heavier the distribution tails are, the higher the positive ACI values will be. For the negative ACI values (in the pit of losses), this statement not always holds. The pit of losses appears

(a)

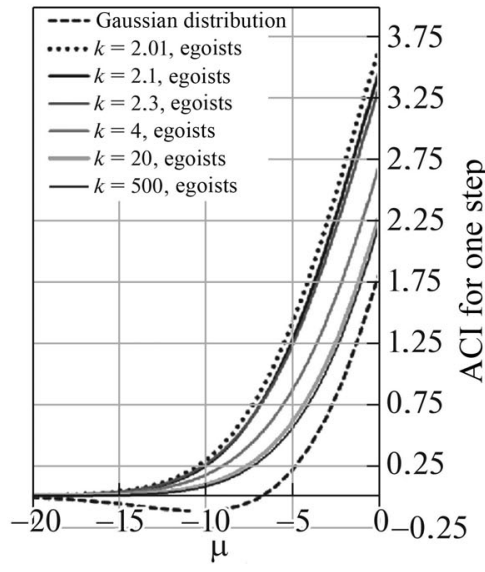

(b)

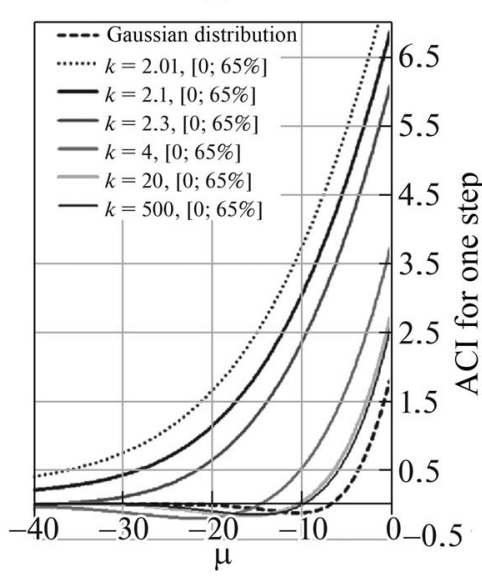

(c)



(d)

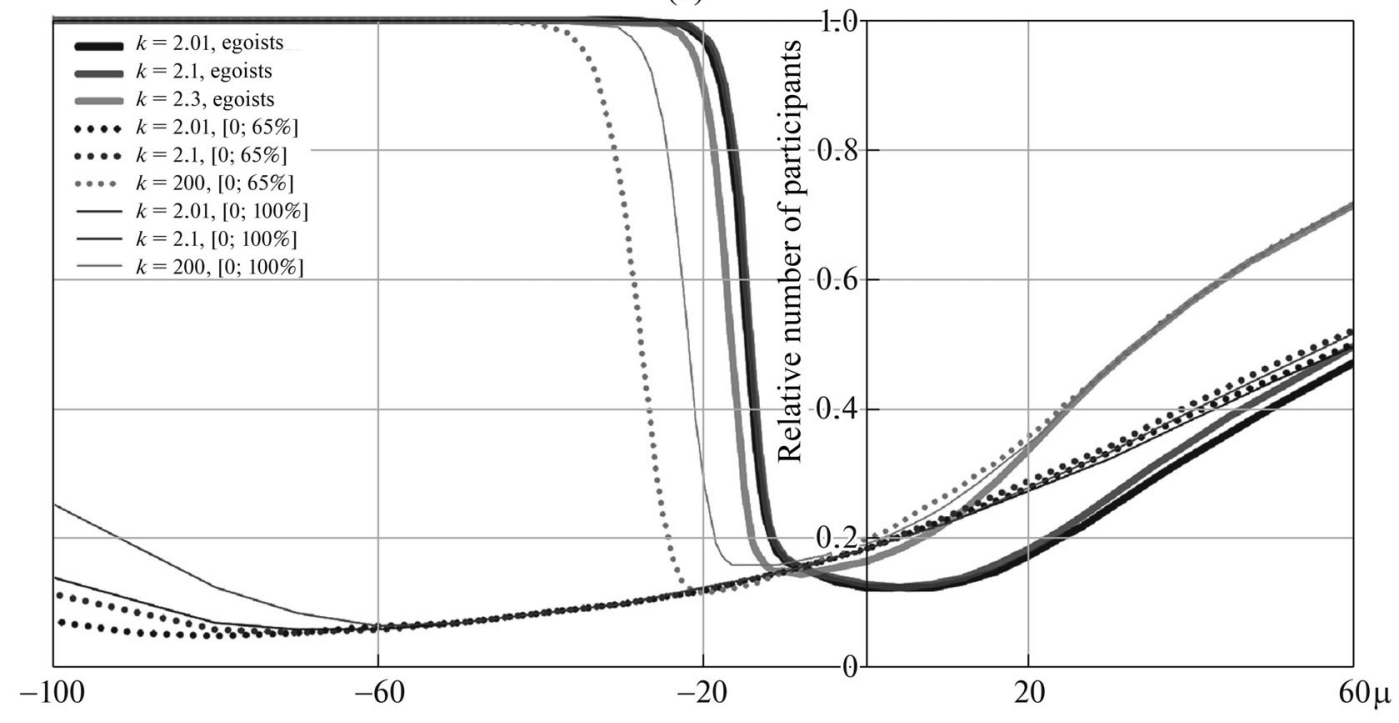

Fig. 5. SP-distributions with $k \in(2,2.3]$ compared to other distributions. ACI for one step without extinction: (a) egoists and (b) altruists with support window [0,65\%]. Egoists and altruists with extinction: (c) ACI for one step and (d) share of participants after 500 steps. Initial number of participants $n=201$; initial capital of each participant $C_{0}=40$; standardization by quartiles; for Gaussian distribution, $\sigma=80$. 
due to the fact that altruists support only $65 \%$ of the society; for the support window [0, 100\%] it is impossible by the definition of an altruistic strategy.

The positive effect of tail heaviness on the growth rate of the ACI is a property manifesting itself precisely under the standardization of distributions by quartiles. Under the meaningfully unjustified standardization by the mean and variance, the opposite regularity is realized (see Fig. 5 in the paper [2]): for $k$ close to 2, the large outliers increasing the ACI have a very low probability, and their presence is compensated by the small typical positive deviations from the mean (see Fig. 1).

In the case of extinction (see Fig. 5c), the heaviness of the tails also increases the ACI, both for egoists and altruists with the support window [0,65\%], affecting much more strongly the ACI of altruists. For a fixed $k$, the ACI of altruists is significantly higher than that of egoists; the ACI of altruists for $k=20$ is comparable to the ACI of egoists for $k=2.1$. Thus, altruistic societies with extinction are more effective than egoistic ones by the ACI criterion.

Figure $5 \mathrm{~d}$ demonstrates the lines of the relative number of participants (in the case of extinction) after 500 voting steps: egoists and altruists with support windows $[0,65 \%]$ and $[0,100 \%]$ with $k$ equal to 2.01, 2.1, and 200. For $\mu=-10$, all the considered relative numbers of participants take values on the small interval $[0.15,0.16]$. For $\mu<-13$ and a fixed $k$, egoists keep their number best of all, then altruists with the support window $[0,100 \%]$ and, finally, altruists with the support window [0,65\%]. Interestingly, for $\mu>-2$ this ranking is reversed! Within the framework of this regularity, differences in numbers in some cases are almost absent, in others they are very large. The fact that for significant negative $\mu$ and symmetrized Pareto distributions, egoists are better at preserving their number than altruists with the support window $[0,100 \%]$, was already noted earlier; see the discussion of Fig. 3b in the paper [2].

For $\mu<-6.6$ the number of egoists is better preserved in the case of heavy-tailed distributions; for $\mu>-6.6$, on the contrary, for distributions with lighter tails. The rates of bankruptcy for distributions with $k=2.01$ and $k=2.1$ differ insignificantly, but for $k=200$ the difference from heavy-tailed distributions becomes considerable.

The heaviness of distribution tails affects the number of altruists negatively, and in a very strong way. For example, for altruists with the support window [0,65\%], $k=200$, and $\mu=-50$, after 500 voting steps the society is almost completely preserved, but in the case $k=2.1$, only $7 \%$ of participants remain. If $\mu>20$, such a society is also much better preserved for high values $k$. Only within the range $-20<\mu<5$ (especially in a slightly unfavorable environment, for $\mu \in[-20,-3]$ ), the parameter $k$ has almost no effect on the final relative number of participants, which grows with increasing $\mu$ from 0.12 to 0.22 . For $\mu \in[-20,-10]$, bankruptcy occurs in an environment with $k=200$ even slightly faster than in environments with $k=2.1$ and $k=2.01$. For altruists with the support window $[0,100 \%]$, on the whole, the same qualitative regularities are observed. Moreover, on the ray $\mu<-10$ the number of participants is preserved better than under the support window $[0,65 \%]$; on the ray $\mu>0$, worse.

Thus, the presence of large outliers appreciably intensifies the process of bankruptcy for altruists, and in the case of sufficiently high $\mu$ (here, if $\mu>-6.6$ ) also for egoists. Under the standardization of distributions by the mean and variance, which leads to a comparison of very dissimilar distributions, this effect takes no place and, on the contrary, the relative number of participants is better preserved for heavy-tailed distributions; see Fig. 5d in the paper [2].

The society goes bankrupt in the fastest way for moderate negative values of the parameter $\mu$. The exception is the societies composed of egoists under distributions with superheavy tails $(k=2.01$ and $k=2.1$ ), which go bankrupt even more intensively in a weakly favorable environment. The deviation of $\mu$ from these values weakens bankruptcy: when $\mu$ decreases, due to the rejection of highest-loss proposals; when $\mu$ increases, due to the higher favor of the proposals. 
(a)

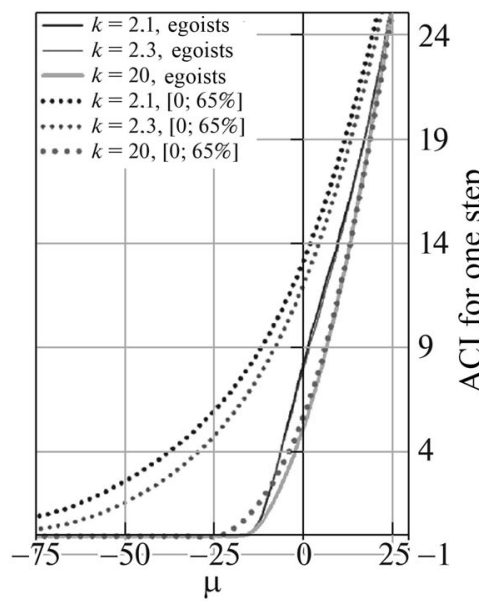

(b)

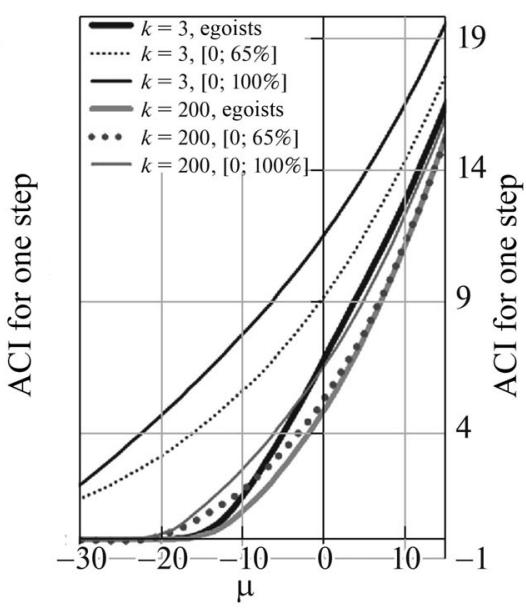

(c)

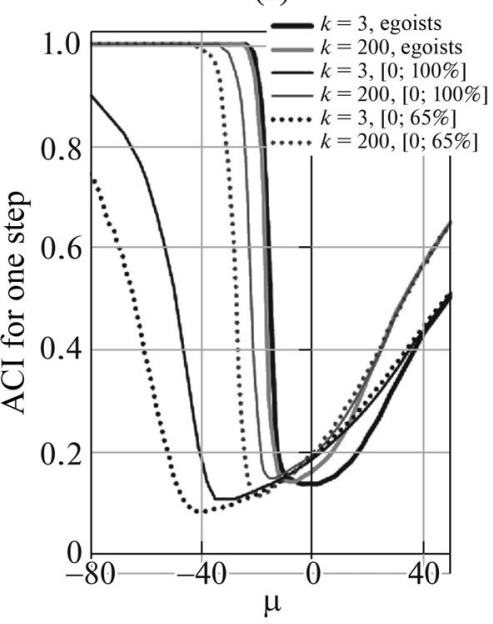

Fig. 6. Characteristics of social dynamics under SP-distributions with extinction; $n=201$; initial capital $C_{0}=40$; standardization by quartiles; distributions from class containing Gaussian distribution with $\sigma=80$. (a), (b) ACI, (c) share of participants after 500 steps.

Comparison of the graphs in Figs. 5c and 5d leads to the following conclusions. The ACI always grows with the weight of the tails, but this growth is accompanied by an increase in the rate of bankruptcy (for altruists, and if $\mu>-6.6$ for egoists as well). The exception is the egoists in the case $\mu<-6.6$ : for this group, a higher heaviness of the tails decelerates the process of bankruptcy simultaneously with the growth of the ACI. However, as $\mu$ increases from these values, the share of accepted proposals goes up. As a result, the frequency of high-damage losses, introduced into the accepted proposals by heavy-tailed distributions, reaches an appreciable level. Therefore, the growth of $\mu$ increases the relative risk of such distributions in terms of bankruptcy.

Figures 6a and 6b show (on a smaller scale than in Fig. 5) the ACI lines of egoists and altruists under negative and positive $\mu$ in the model with extinction. In the case $\mu>0$, the ACI also grows with decreasing $k$, i.e., for higher heaviness of the tails.

In a favorable environment (as well as in a moderately unfavorable one), altruists with the support window $[0,100 \%]$ have the highest ACI; the ACI of altruists with the support window $[0,65 \%]$ is next by value; the ACI of egoists is even lower. This ranking is, of course, independent of the standardization of distributions, since it does not require comparison of distributions with each other.

The graphs of the number of participants on additional data (see Fig. 6c, where the SPdistribution with $k=3$ is also presented) confirm the conclusions obtained in the analysis of Fig. 5d: under heavy-tailed distributions, the society is preserved worse (the exception is the society composed of the egoists in the case $\mu<-6$ ), achieving however a higher value of the ACI of the participants. Note that the residual numbers of altruists and egoists are close to one another in mildly unfavorable and favorable $(\mu>-12)$ environments under relatively light $(k=200)$ tails of the distribution used to generate proposals.

In Fig. 6a, the graphs of egoistic societies for $k=2.1$ and $k=2.3$ are almost the same. Within the presented range of $\mu$, the ACI of altruists with the support window [0,65\%] and the same $k$ is positive and significantly higher than the ACI of egoists. The latter is almost indistinguishable from 0 for $\mu<-15$, and positive for larger values of $\mu$. In the case $k=20$, the difference between the ACI graphs of altruists with the support window $[0,65 \%]$ and egoists becomes much smaller: the former are noticeably different from (and ahead of) the latter only for $-23<\mu<5$, and for $\mu<-23$ the ACI of both is close to 0 . Altruists do not outrun egoists more significantly, in particular, due to the 
fact that under the support window [0,65\%], they ignore capital increments of $35 \%$ of participants. The general simple conclusion is as follows: heavy distribution tails increase the ACI of both egoists and altruists, but considerably stronger for the latter. A list of key conclusions will be provided in Section 7 .

\section{LOGISTIC DISTRIBUTION AND STUDENT'S $t$-DISTRIBUTION}

Section 5 has presented an analysis of the capital dynamics in the case of proposals generated using the Gaussian distribution and symmetrized Pareto distributions. These two generators are contrasted in two ways: the classical bell-shaped distribution with light $\left(\sim e^{-x^{2}}\right)$ tails and the heavy-tailed distribution with a sharp peak. Of course, the class of symmetric unimodal distributions on the real line includes a rich variety of representatives with different properties. To assess the influence of the type of distribution on the capital dynamics, consider also Student's $t$-distribution with 3 degrees of freedom (for 2 degrees of freedom, this distribution has no variance) and the logistic distribution. The corresponding characteristics of social dynamics in comparison with the distributions considered earlier are shown in Fig. 7.

For a society composed of egoists (Fig. 7a), all the distributions under consideration with $\mu<0$ are uniquely ranked by the ACI: for the Gaussian distribution, the ACI is the lowest, followed by the logistic one; the latter is noticeably inferior to Student's t distribution; finally, the symmetrized Pareto distributions with $k=500$ and $k=20$ demonstrate the best results, leading to approximately the same ACI values. This ranking coincides with the ranking by the heaviness of tails. Thus, the added distributions confirm the regularities revealed in the analysis of Fig. 5a.

In the case of a society composed of altruists with the support window [0,65\%], the regularity is somewhat more complicated; see Fig. 7b. The ACI lines $(\mu)$ for the logistic distribution and the Student's $t$-distribution can be obtained from the corresponding line for the Gaussian distribution by successive shifts to the left and slightly downward, in combination with small additional stretches. Thus, for $\mu$ smaller by several unities, the ACI values are somewhat lower than for the Gaussian distribution. As a result, an increase in the heaviness of tails leads to an increase in the ACI only for positive and relatively small (by absolute value) negative $\mu$; with a further decrease in $\mu$ it is more beneficial that the distribution tails be lighter. The ACI under the Student's $t$-distribution and the SP-distributions with $k=20$ and $k=500$, like in a society composed of egoists, are quite close, but in this case the Student's $t$-distribution with 3 degrees of freedom is a little more similar to the SP-distribution with $k=20$ in terms of the ACI.

In the model with extinction (Fig. 7c), the logistic distribution has the same feature as the Gaussian distribution: the ACI lines of egoists and altruists with the support window [0,65\%] are very close to one another, but the logistic distribution yields slightly greater values of the ACI. The Student's $t$-distribution for altruists with the support window [0,65\%] gives a higher gain in comparison with the logistic distribution, but for egoists this gain is smaller. Thus, under the Student's t-distribution, it is much more beneficial to the participants (in the sense of the ACI) to be altruists with the window $[0,65 \%]$ than to be egoists, and this peculiarity brings the abovementioned distribution closer to Pareto distributions. It is closest to the SP-distribution with $k=20$ : the difference in their capital increment curves for both types of societies is insignificant. Also, note a subtle effect as follows: for considerable negative values of $\mu$, egoists have a higher ACI when proposals are generated using the Student's $t$-distribution with 3 degrees of freedom, but for $\mu>-5$ it turns out to be more beneficial to them to deal with the Pareto distribution with $k=20$. Similarly, for altruists with the support window [0,65\%], the Pareto distribution with $k=20$ reaches almost the same ACI dynamics in the case $\mu=-1$ as the Student's $t$-distribution.

Figure $7 d$ shows the ACI lines of altruists with the support windows $[0,65 \%]$ and $[0,100 \%]$ under the Student's $t$-distribution, logistic distribution and SP-distributions with $k=200$. Under each of 
(a)



(d)

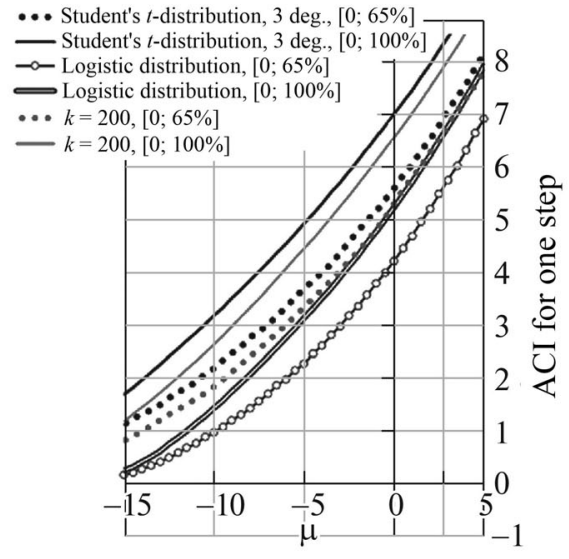

(b)

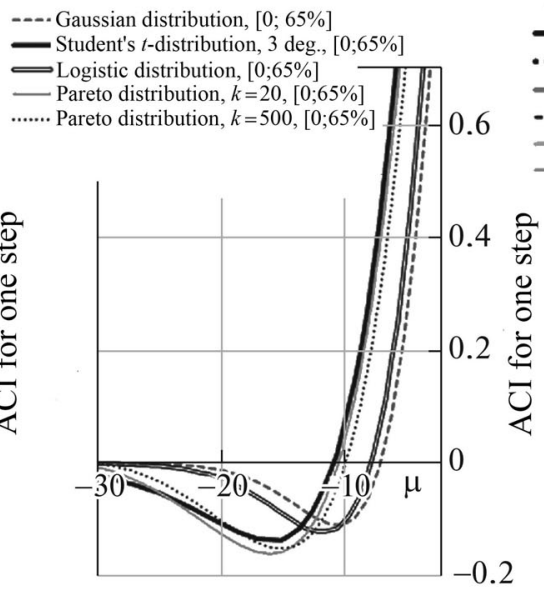

(c)

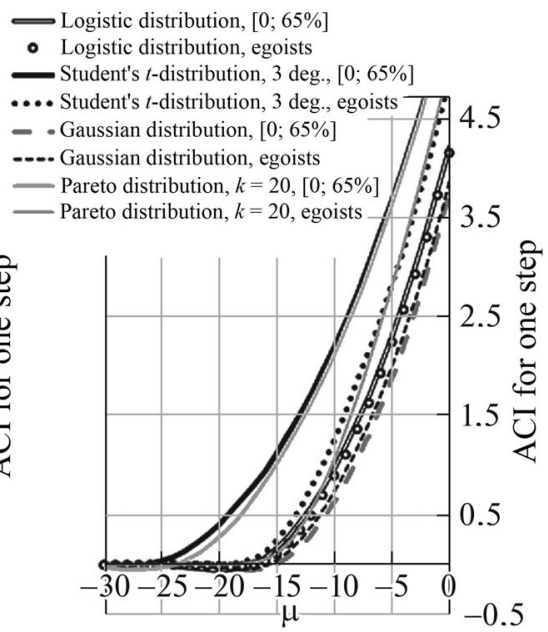

(e)

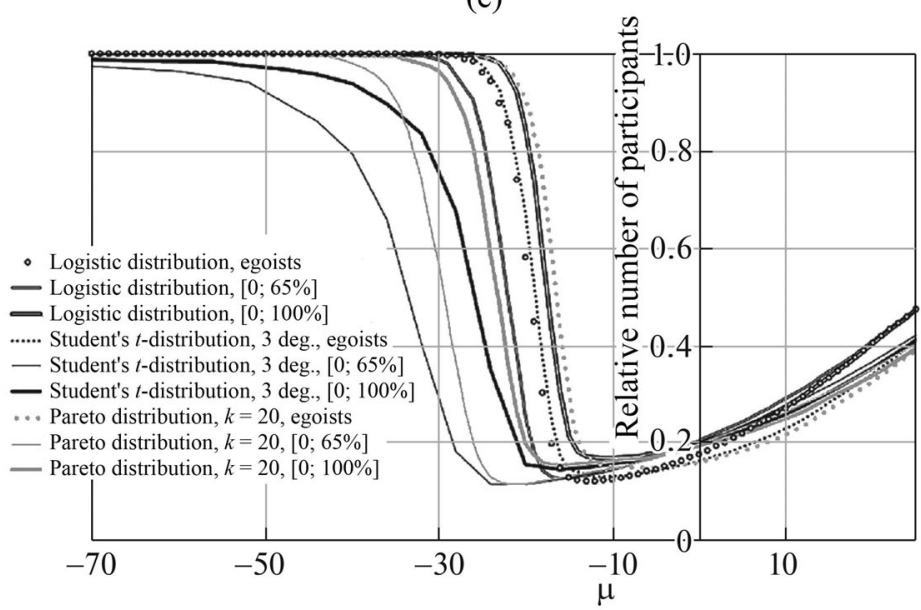

Fig. 7. Logistic distribution and Student's $t$-distribution with 3 degrees of freedom compared to other distributions. ACI for one step in model without extinction: (a) egoists, (b) altruists with support window [0, 65\%]. Egoists and altruists in model with extinction: (c), (d) ACI for one step, (e) share of participants after 500 steps given participant's initial capital $C_{0}=40$. Initial number of participants $n=201$; standardization by quartiles; for Gaussian distribution, $\sigma=80$.

them, the wider support window leads to larger values of the ACI, and the gap increases with $\mu$. In other words, the more favorable the environment within the considered range of $\mu$ is, the less rational (in terms of the ACI criterion) the support of only $65 \%$ of the poorest will be, compared to the support of the entire society. The SP-distribution with $k=20$ leads to approximately the same results as the Student's $t$-distribution with 3 degrees of freedom (see Fig. 7c). At the same time, under the SP-distribution with $k=200$, which has lighter tails, the ACI of both egoists and altruists is smaller.

The residual numbers of participants are shown in Fig. 7e. Note that the lines of egoists under the Student's $t$-distribution and the logistic distribution with $\mu<0$ are very close to one another; for $\mu>0$, higher values are given by the logistic distribution. In the case of the Student's $t$-distribution, for sufficiently large (by absolute value) negative $\mu$, the egoists preserve their number better than the altruists; in the case of the logistic distribution, the number of egoists lies between the numbers of altruists with the support windows $[0,65 \%]$ and $[0,100 \%]$. Thus, here again, the Student's $t$-distribution demonstrates the same qualitative properties as the Pareto distribution, 
and the logistic distribution only slightly deviates from the Gaussian one towards heavy-tailed distributions. (Under the Gaussian distribution, the number of egoists and altruists with the support window $[0,65 \%]$ are close to one another; see Fig. 3a in the paper [2].)

In the model with extinction, the Student's $t$-distribution and Pareto distribution with $k=20$ lead to similar results in terms of the ACI (Fig. 7c). However, concerning the number of participants, the Pareto distribution is appreciably more beneficial to both egoists and altruists in a rather unfavorable environment, and the Student's $t$-distribution is somewhat more beneficial to them in a considerably favorable environment. In a neutral environment (and for altruists, if $-4<\mu<10$ ), the residual numbers of participants under these two distributions almost coincide with one another. For $\mu<-4$, see the discussion above, nearly the same residual numbers of participants are obtained under the Student's $t$-distribution and the logistic distribution.

For egoists, in the case $\mu<-4$, the SP-distribution is most beneficial in terms of the number of participants; in the case $\mu>-4$, the logistic distribution. For altruists, the logistic distribution is most beneficial regardless of $\mu$; however, for $-10<\mu<5$, the Student's $t$-distribution and Pareto distributions yield almost the same numbers of participants.

For $\mu<-4$, the general leadership in terms of the number of participants belongs to egoists under the Pareto distribution. For positive values of $\mu$, they are in last place, and the first place is occupied by altruists with the support window $[0,65 \%]$ in a stochastic environment with logistically distributed proposals. For $\mu=20$, they are approached by both altruists with the support window $[0,100 \%]$ and egoists, in the case of the logistic distribution as well.

\section{MAIN RESULTS}

The main (and simplest) results, established in this paper by studying the dynamics of the ViSE model, include the following.

1. When standardized by quartiles, the heaviness of the distribution tails increases the ACI of both egoists and altruists, in the cases of extinction and no extinction. Note that the ACI of altruists increases significantly more, but with one exception: for considerable negative values of the parameter $\mu$, an increase in the heaviness of the tails can lead not to an increase in the ACI of altruists with the support window [0,65\%], but to a shift of the pit of losses to the left and even to its deepening.

2. In the case of extinction, the heaviness of the distribution tails accelerates the bankruptcy of both egoists and altruists, but also with one exception: for considerable negative values of the parameter $\mu$, the bankruptcy of egoists under heavy-tailed distributions, on the contrary, slows down somewhat. Thus, an increase in one criterion (ACI) under varying distribution parameters is often accompanied by a decrease in the other (the number of participants).

3. The logistic distribution in the ViSE model gives results that are moderately different from the Gaussian distribution towards the distributions with heavier tails.

4. The Student's $t$-distribution with 3 degrees of freedom is similar in terms of the ACI to the SPdistribution with $k=20$, especially for egoists and altruists with the support window [0,65\%] in the model with extinction. In the case of no extinction, for egoists, the ACI under the Student's $t$-distribution is approximately the same as under the distributions with lighter tails (even smaller than under the SP-distributions with $k=500$ ); for altruists with the support window $[0,65 \%]$, on the contrary, the ACI is like under one of the SP-distributions with heavier tails than for $k=20$. The latter regularity becomes even more pronounced for the graphs of the residual number of participants. For egoists, the Student's $t$-distribution curve lies between the curves of the logistic and SP-distributions, almost matching the former for considerable negative values of the parameter $\mu$ and almost matching the latter for $\mu>0$. For altruists with 
the support windows $[0,65 \%]$ and $[0,100 \%]$, the Student's $t$-distribution gives values typical of distributions with heavier tails than the SP-distribution with $k=20$. This difference between the curves may be due to the difference in shape: a sharp peak in the SP-distribution and the bell-shaped form of the Student's $t$-distribution.

A number of other regularities has been discussed in Sections 4 and 5.

\section{CONCLUSIONS}

This paper has studied the dynamics of homogeneous societies within the ViSE model with proposals generated using distributions that differ in the heaviness of tails: the Gaussian distribution, the logistic distribution, the Student's t-distribution, and the symmetrized Pareto distributions. In [2] it was found that the heaviness of the tails of the distribution of random variables used to generate proposals significantly affects the capital dynamics and the effectiveness of the strategies of different participants. For a quantitative analysis of the influence of this factor, it is necessary to introduce classes of distributions that differ in it and are similar in other essential parameters. In [2], such classes were constructed by unifying the mean $\mu$ and variance $\sigma^{2}$, which led to the consideration of distributions with heavy tails, for which the probability of significant deviations (in the scale of $\sigma$ ) from the average is too low. This makes the standard deviation a counterintuitive measure of scatter in the case of super-heavy tailed distributions and the research of the present type.

In this paper, the scatter has been estimated using quartiles. As a result, each class of comparable distributions includes the ones whose densities are moderately different on the interval where the major part of the probability is concentrated. With this approach to the unification of distributions, it has been established that the heavier distribution tails (meaning the overflow of probability from average outliers to larger ones, and also, possibly, to observations close to the average) leads to higher rates of bankruptcy, but at the same time allows participants to accumulate higher capital through the implementation of collective decisions. The exception is a society composed of altruists in an unfavorable environment, for which an increase in the heaviness of the distribution tails can lead to a shift of the "pit of losses" to the left and down. Also, a number of more subtle effects has been established that characterize the capital dynamics of homogeneous societies with different social attitudes of participants in different stochastic environments.

\section{REFERENCES}

1. Chebotarev, P.Yu., Analytical Expression of the Expected Values of Capital at Voting in the Stochastic Environment, Autom. Remote Control, 2006, vol. 67, no. 3, pp. 480-492.

2. Chebotarev, P.Yu., Tsodikova, Ya.Yu., Loginov, A.K., Lezina, Z.M., Afonkin, V.A., and Malyshev, V.A., Comparative Efficiency of Altruism and Egoism as Voting Strategies in Stochastic Environment, Autom. Remote Control, 2018, vol. 79, no. 11, pp. 2052-2072.

3. Mirkin, B.G., Problema gruppovogo vybora, Moscow: Nauka, 1974. Translated under the title Group Choice, Fishburn, P.C., Ed., New York: Wiley, 1979.

4. Plott, C.R., A Notion of Equilibrium and Its Possibility under Majority Rule, Am. Econ. Rev., 1967, vol. 58, no. 4, pp. 787-806.

5. McKelvey, R.D., Intransitivities in Multidimensional Voting Models and Some Implications for Agenda Control, J. Economic Theory, 1976, vol. 12, no. 3, pp. 474-482.

6. Schofield, N., The Spatial Model of Politics, London: Routledge, 2007.

7. Chebotarev, P.Yu., Some Properties of Paths in the Dynamic Voting Problem, Autom. Remote Control, 1986, vol. 47, no. 1, part 2, pp. 121-126.

AUTOMATION AND REMOTE CONTROL Vol. $81 \quad$ No. 102020 
8. Saari, D.G., The Generic Existence of a Core for q-rules, Economic Theory, 1997, vol. 9, no. 2, pp. 219260.

9. Novikov, S.G., One Dynamic Problem in Voting Theory. I, Autom. Remote Control, 1985, vol. 46, no. 8, pp. 1016-1026.

10. Novikov, S.G., One Dynamic Problem in Voting Theory. II, Autom. Remote Control, 1985, vol. 46, no. 9, pp. 1168-1177.

11. McKelvey, R.D., Game Theoretic Models of Voting in Multidimensional Issue Spaces, in Game Theory Appl., Ichiishi, T., Neyman, A., and Tauman, Y., Eds., San Diego: Academic, 1990, pp. 317-335.

12. Ordeshook, P.C., The Spatial Analysis of Elections and Committees: Four Decades of Research, in Perspective on Public Choice: A Handbook, Mueller, D., Ed., Cambridge: Cambridge Univ. Press, 1997, pp. 247-270.

13. Lehtinen, A., The Welfare Consequences of Strategic Voting in Two Commonly Used Parliamentary Agendas, Theor. Decis., 2007, vol. 63, no. 1, pp. 1-40.

14. Kress, D. and Pesch, E., Sequential Competitive Location on Networks, Eur. J. Oper. Res., vol. 217, no. 3, pp. 483-499.

15. Chebotarev, P.Yu., Malyshev, V.A., Tsodikova, Ya.Yu., Loginov, A.K., Lezina, Z.M., and Afonkin, V.A., The Optimal Majority Threshold as a Function of the Variation Coefficient of the Environment, Autom. Remote Control, 2018, vol. 79, no. 4, pp. 725-736.

16. Chebotarev, P.Yu., Loginov, A.K., Tsodikova, Ya.Yu., Lezina, Z.M., and Borzenko, V.I., Analysis of Collectivism and Egoism Phenomena within the Context of Social Welfare, Autom. Remote Control, 2010, vol. 71, no. 6, pp. 1196-1207.

17. Chebotarev, P.Yu., Loginov, A.K., Tsodikova, Ya.Yu., et al., "The Snowball of Cooperation" and "Snow Communism," Tr. IV mezhd. konf. po problemam upravleniya (Proc. 4th Int. Conf. on Control Problems), Moscow: Inst. Probl. Uprvalen., 2009, pp. 687-699.

18. Malyshev, V., Optimal Majority Threshold in a Stochastic Environment, arXiv preprint math.OC/1901. 09233, 2019.

19. Tsodikova, Y., Chebotarev, P., Loginov, A., and Lezina, Z., Modeling Responsible Elite, in Recent Advances of the Russian Operations Research Society, Aleskerov, F. and Vasin, A., Eds., Newcastle upon Tyne: Cambridge Scholars Publishing, 2020, pp. 89-110. arXiv preprint physics.soc-ph/1906.02072, 2019.

20. Chebotarev, P.Yu., Tsodikova, Ya.Yu., Loginov, A.K., and Lezina, Z.M., What Percentage of Altruists is Needed in a Society, and Where Should They Focus Their Efforts?, Tr. II Ross. ekonomicheskogo kongressa (Proc. 2nd Russian Economic Congress), Suzdal, February 18-22, 2013, Moscow: New Economic Association, 2013, pp. 1-3. http://skachate.ru/download/filosofiya-31943/31943.doc

21. Wilson, E.O., On Human Nature, Cambridge: Harvard Univ. Press, 1979.

22. Chebotarev, P.Yu., How to Build a Civil Society Using a Scientific Approach, Troitskii Variant-Nauka, 2015, no. 186, pp. 1-2. http://trv-science.ru/uploads/186N.pdf

23. Groeneveld, R.A., A Class of Quantile Measures for Kurtosis, Am. Statistician, 1998, vol. 52, no. 4, pp. $325-329$.

24. Arriaza, A. et al., Shape Measures Based on the Convex Transform Order, Metrika, 2019, vol. 82, no. 1, pp. 99-124.

25. Brys, G., Hubert, M., and Struyf, A., Robust Measures of Tail Weight, Comput. Stat. Data Anal., 2006, vol. 50, no. 3, pp. 733-759.

26. Hinkley, D.V., On Power Transformations to Symmetry, Biometrika, 1975, vol. 62, pp. 101-111.

This paper was recommended for publication by F.A. Aleskerov, a member of the Editorial Board 